\title{
Influence of confining potentials on the exchange coupling in double quantum dots
}

Pedersen, Jesper Goor; Flindt, Christian; Jauho, Antti-Pekka; Mortensen, Asger

Published in:

Physical Review B Condensed Matter

Link to article, DOI:

10.1103/PhysRevB.81.193406

Publication date:

2010

Document Version

Publisher's PDF, also known as Version of record

Link back to DTU Orbit

Citation (APA):

Pedersen, J. G., Flindt, C., Jauho, A-P., \& Mortensen, A. (2010). Influence of confining potentials on the exchange coupling in double quantum dots. Physical Review B Condensed Matter, 81(19), 193406.

https://doi.org/10.1103/PhysRevB.81.193406

\section{General rights}

Copyright and moral rights for the publications made accessible in the public portal are retained by the authors and/or other copyright owners and it is a condition of accessing publications that users recognise and abide by the legal requirements associated with these rights.

- Users may download and print one copy of any publication from the public portal for the purpose of private study or research.

- You may not further distribute the material or use it for any profit-making activity or commercial gain

- You may freely distribute the URL identifying the publication in the public portal 


\title{
Influence of confining potentials on the exchange coupling in double quantum dots
}

\author{
Jesper Goor Pedersen, ${ }^{1}$ Christian Flindt, ${ }^{2}$ Antti-Pekka Jauho, ${ }^{3,4}$ and Niels Asger Mortensen ${ }^{1}$ \\ ${ }^{1}$ DTU Fotonik, Department of Photonics Engineering, Technical University of Denmark, Building 343, \\ DK-2800 Kongens Lyngby, Denmark \\ ${ }^{2}$ Department of Physics, Harvard University, 17 Oxford Street, Cambridge, Massachusetts 02138, USA \\ ${ }^{3}$ DTU Nanotech, Department of Micro and Nanotechnology, Technical University of Denmark, Building 345 East, \\ 2800 Kongens Lyngby, Denmark \\ ${ }^{4}$ Department of Applied Physics, Aalto University, P.O. Box 11100, FI-00076 Aalto, Finland \\ (Received 17 February 2010; revised manuscript received 14 April 2010; published 17 May 2010)
}

\begin{abstract}
We report simple expressions for the exchange coupling in double quantum dots calculated within the Heitler-London and the Hund-Mulliken approximations using four different confining potentials. At large interdot distances and at large magnetic fields, the exchange coupling does not depend significantly on the details of the potentials. In contrast, at low fields and short distances, different behaviors of the exchange coupling can be attributed to particular features of the potentials. Our results may be useful as guidelines in numerical studies and in the modeling of experiments.
\end{abstract}

DOI: 10.1103/PhysRevB.81.193406

PACS number(s): 73.21.La, 75.30.Et

\section{INTRODUCTION}

The exchange coupling between electron spins in tunnel coupled quantum dots constitutes a key element in proposals for implementing quantum information processing in the solid state. ${ }^{1,2}$ The exchange coupling splits the singlet and triplet spin states, depending on the confining potential and the applied magnetic field, thereby enabling electrical (or magnetic) control of the exchange coupling as demonstrated in recent experiments. ${ }^{3}$ The ability to control the exchange coupling with external fields, however, also makes the exchange coupling susceptible to electromagnetic fluctuations in the environment. A current trend is thus to search for "sweet spots" in parameter space, ${ }^{4-7}$ i.e., local maxima of the exchange coupling as function of external fields, where the exchange coupling to first order is insensitive to fluctuations.

Calculations of the exchange coupling can be approached with a variety of analytic and numerical methods. These include several analytic approximations ${ }^{2}$ and numerical schemes such as exact diagonalization, ${ }^{8-11}$ path integral Monte Carlo simulations, ${ }^{12}$ and configuration interaction calculations combined with density functional theory. ${ }^{6}$ Numerical methods allow for calculations of the exchange coupling with high precision. However, in order to gain an understanding of the dependence of the exchange coupling on different parameters or as guidelines in the search for sweet spots, ${ }^{4-7}$ closed-form analytic expressions can be very useful.

In this Brief Report, we present simple analytic expressions for the exchange coupling of a double quantum dot obtained within the Heitler-London and Hund-Mulliken approximations $^{2}$ for four different confining potentials. We provide a comparative study of the exchange coupling calculated analytically for the four potentials as functions of interdot distance and magnetic field. In particular, we identify certain properties that are only weakly dependent on the choice of potential and discuss other features that, in contrast, can be associated with particular details of the potentials.

\section{MODEL}

We consider two electrons confined by a double quantum dot in two dimensions in a perpendicular magnetic field. The two-electron Hamiltonian is

$$
H\left(\mathbf{r}_{1}, \mathbf{r}_{2}\right)=h\left(\mathbf{r}_{1}\right)+h\left(\mathbf{r}_{2}\right)+C\left(\left|\mathbf{r}_{1}-\mathbf{r}_{2}\right|\right),
$$

where

$$
C\left(\left|\mathbf{r}_{1}-\mathbf{r}_{2}\right|\right)=\frac{e^{2}}{4 \pi \varepsilon_{r} \varepsilon_{0}\left|\mathbf{r}_{1}-\mathbf{r}_{2}\right|}
$$

is the Coulomb interaction and the single-particle Hamiltonian in the effective-mass approximation is

$$
h(\mathbf{r})=\frac{1}{2 m}[\mathbf{p}+e \mathbf{A}(\mathbf{r})]^{2}+V(\mathbf{r}), \quad \mathbf{r}=(x, y) .
$$

The confining potential is denoted as $V(\mathbf{r}), m$ is the effective electron mass, and $\mathbf{A}(\mathbf{r})=B_{z}(-y, x) / 2$ the magnetic vector potential. The Zeeman splitting does not affect the exchange coupling and has been omitted above. We use parameters typical of GaAs and take $m=0.067 m_{e}$ and $\varepsilon_{r}=12.9$. The exchange coupling $J_{V}\left(B_{z}\right)=E_{T}-E_{S}$ is the difference between the spin-triplet and spin-singlet orbital ground states, respectively.

The four potentials considered in this work are defined in Fig. 1 with $r_{0}=\sqrt{\hbar / m \omega_{0}}$ being the oscillator length, $\hbar \omega_{0}$ the confinement energy, and $2 d$ the center to center distance between the dots. The first three potentials have previously been considered in the literature. ${ }^{2,5,7-13}$ The following approximations take as starting point the uncoupled dots at large distances, $d \gg r_{0}$. For the left or right dot centered at $\mathbf{r}_{L / R}=(\mp d, 0)$, the ground state can be written ${ }^{14}$ as $\varphi_{\mp d}(x, y)=\langle\mathbf{r} \mid L / R\rangle=e^{ \pm i y d / 2 l_{B}^{2}} \varphi(x \mp d, y)$ in terms of the FockDarwin ground state $\varphi(x, y)=\sqrt{\frac{m \omega}{\pi \hbar}} e^{-m \omega\left(x^{2}+y^{2}\right) / 2 \hbar}$. Here, the magnetic length is $l_{B}=\sqrt{\hbar / e B_{z}}$ and $\omega=b \omega_{0}$, where $b$ $=\sqrt{1+\omega_{L}^{2} / \omega_{0}^{2}}$ is the magnetic compression factor and $\omega_{L}$ $=e B_{z} / 2 m$ the Larmor frequency. Additionally, we shall need the overlap $S \equiv\langle L \mid R\rangle=e^{-\tilde{d}^{2}(2 b-1 / b)}$, where $\tilde{d}=d / r_{0}$. 

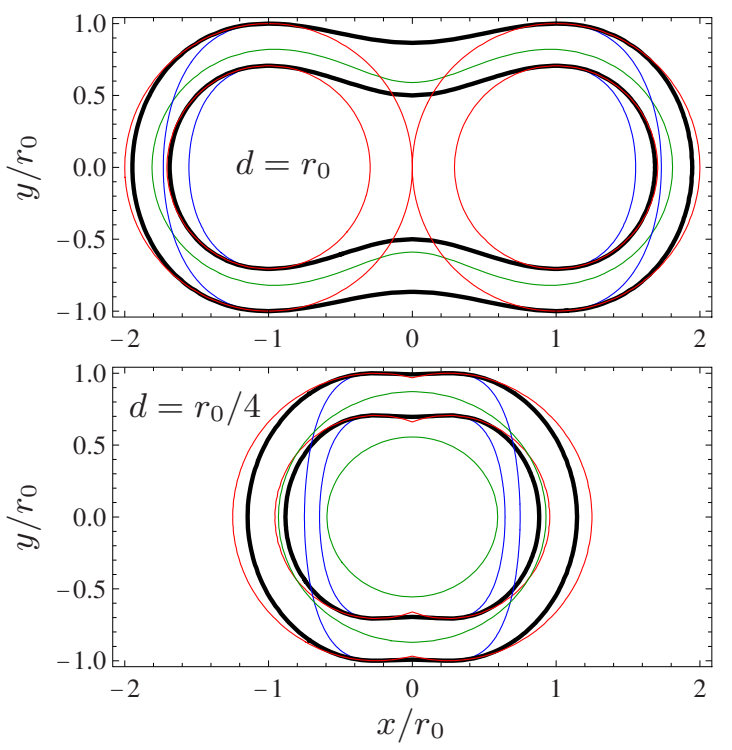

$-v_{\mathrm{q}}(x, y)=\frac{\left(x^{2}-d^{2}\right)^{2}}{4 d^{2}}+y^{2}$

$-v_{\min }(x, y)=\min \left\{(x-d)^{2},(x+d)^{2}\right\}+y^{2}$

$-v_{\exp }(x, y)=-r_{0}^{2} e^{-\left\{(x-d)^{2}+y^{2}\right\} / r_{0}^{2}}$

$-r_{0}^{2} e^{-\left\{(x+d)^{2}+y^{2}\right\} / r_{0}^{2}}$

$-v_{\mathrm{q}}^{\prime}(x, y)=\frac{\left(x^{2}-d^{2}\right)^{2}}{4 d^{2}+x^{2}}+y^{2}$

FIG. 1. (Color online) Contour plots of the model potentials at two different interdot distances. The potentials are $V_{i}(x, y)$ $\equiv\left(m \omega_{0}^{2} / 2\right) v_{i}(x, y)$. The contours correspond to $v(x, y)=r_{0}^{2} / 4$ and $v(x, y)=r_{0}^{2} / 2$. The potential $v_{\exp }$ has been shifted such that $v_{\exp }( \pm d, 0)=0$.

\section{HEITLER-LONDON}

Within this approximation, the exchange coupling is estimated as

$$
J^{(\mathrm{HL})}=\langle-|H|-\rangle-\langle+|H|+\rangle,
$$

where $| \pm\rangle=(|L\rangle|R\rangle \pm|R\rangle|L\rangle) / \sqrt{2\left(1 \pm S^{2}\right)}$. The exchange coupling is composed of contributions $J^{(\mathrm{HL})}=J_{C}^{(\mathrm{HL})}+J_{h}^{(\mathrm{HL})}$ from the Coulomb interaction and from the single-particle Hamiltonians, respectively. The first term $J_{C}^{(\mathrm{HL})}$ does not depend on the potential and is given by Eq. (7) in Ref. 2. The second term $J_{h}^{(\mathrm{HL})}$ is listed in Table I for each of the four potentials.
The result for $V_{q}$ has previously been reported in Ref. 2. The Heitler-London approximation is typically reliable when the ratio $\frac{e^{2}}{4 \pi \epsilon_{r} \epsilon_{0} r_{0}} / \hbar \omega_{0}$ is small. ${ }^{13}$

\section{HUND-MULLIKEN}

The Heitler-London approximation considers only the singly occupied singlet and triplet states. When the tunnel coupling between the quantum dots becomes large, the doubly occupied spin-singlet states should also be taken into account. The exchange coupling is then obtained by diagonalizing the Hamiltonian in the Hilbert space spanned by $\Psi_{ \pm d}^{D}\left(\mathbf{r}_{1}, \mathbf{r}_{2}\right)=\Phi_{ \pm d}\left(\mathbf{r}_{1}\right) \Phi_{ \pm d}\left(\mathbf{r}_{2}\right) \quad$ and $\quad \Psi_{ \pm}^{S}\left(\mathbf{r}_{1}, \mathbf{r}_{2}\right)$ $=\left[\Phi_{+d}\left(\mathbf{r}_{1}\right) \Phi_{-d}\left(\mathbf{r}_{2}\right) \pm \Phi_{-d}\left(\mathbf{r}_{1}\right) \Phi_{+d}\left(\mathbf{r}_{2}\right)\right] / \sqrt{2}$, where $\Phi_{ \pm d}$ are the orthonormalized single-particle states $\Phi_{ \pm d}=\left(\varphi_{ \pm d}\right.$ $\left.-g \varphi_{\mp d}\right) / \sqrt{1-2 S g+g^{2}}$, with $g=\left(1-\sqrt{1-S^{2}}\right) / S$. The exchange coupling is now estimated as ${ }^{2}$

$$
J^{(\mathrm{HM})}=V-U_{r} / 2+\frac{1}{2} \sqrt{U_{r}^{2}+16 t_{r}^{2}} .
$$

Here, $U_{r}$ and $t_{r}=t-w=-\left\langle\Phi_{ \pm d}|h| \Phi_{\mp d}\right\rangle-\left\langle\Psi_{+}^{S}|C| \Psi_{ \pm d}^{D}\right\rangle / \sqrt{2}$ are the renormalized on-site Coulomb interaction and tunnel coupling, respectively, and $V$ (not to be confused with the confining potential) is the difference in Coulomb energy between the singly occupied singlet and triplet states. We find that the bare tunnel coupling can be written as $t$ $=J_{h}^{(\mathrm{HL})}\left(1+S^{2}\right) / 4 S$, where $J_{h}^{(\mathrm{HL})}$ is given in Table I and $U_{r}, w$, and $V$ can be found in Appendix A of Ref. 2.

\section{RESULTS}

In Fig. 2, we show the exchange coupling $J^{(\mathrm{HL})}$ in the Heitler-London approximation at zero magnetic field as function of the interdot distance. We note that the exchange coupling at zero magnetic field always must be nonnegative. At large distances, $d>r_{0}$, the potentials separate into two isolated quantum dots with qualitatively similar behavior for the exchange coupling, showing a decay with increasing interdot distance. The potential $V_{\min }$ results in a slightly lower exchange coupling compared to the other potentials at a given large interdot distance. At short distances, in contrast, the results depend strongly on the particular choice of potential. For both confinement energies, the potential $V_{q}$ yields nonnegative results. However, at short distances, $d \ll r_{0}$, the exchange coupling for this potential diverges. In contrast, the

TABLE I. Contribution $J_{h}^{(\mathrm{HL})}$ to the exchange coupling within the Heitler-London approximation for the four potentials. The complementary error function is denoted as $\operatorname{erfc}(x)$, and $\tilde{d}=d / r_{0}$.

\begin{tabular}{lc}
\hline \hline & $J_{h}^{(\mathrm{HL})} /\left(\hbar \omega_{0}\right)$ \\
\hline$V_{q}$ & $\frac{2 S^{2}}{1-S^{4}} \frac{3}{4 b}\left(1+b \tilde{d}^{2}\right)$ \\
$V_{\min }$ & $\frac{2 S^{2}}{1-S^{4}}\left[\frac{2 \tilde{d}}{\sqrt{b \pi}}\left\{1-e^{-b \tilde{d}^{2}}\right\}+2 \tilde{d}^{2} \operatorname{erfc}(\tilde{d} \sqrt{b})\right]$ \\
$V_{\exp }$ & $\left.\frac{2 S^{2}}{1-S^{4}} \frac{\tilde{d}^{2}}{b^{2}}-\frac{b}{1+b}\left\{1+e^{-4 b \tilde{d}^{2} /(1+b)}-2 e^{-\tilde{d}^{2} /\left(b^{2}+b\right)}\right\}\right]$ \\
$V_{q}^{\prime}$ & $\frac{2 S^{2}}{1-S^{4}}\left[2 \tilde{d}^{2}-\frac{25}{2} \sqrt{b \pi} \widetilde{d}^{3}\left(e^{4 b \tilde{d}^{2}} \operatorname{erfc}(2 \sqrt{b} \tilde{d})-\operatorname{Re}\left\{e^{(3+4 i) \tilde{d}^{2}} \operatorname{erfc}[(2+i) \sqrt{b} \tilde{d}]\right\}\right)\right]$ \\
\hline
\end{tabular}




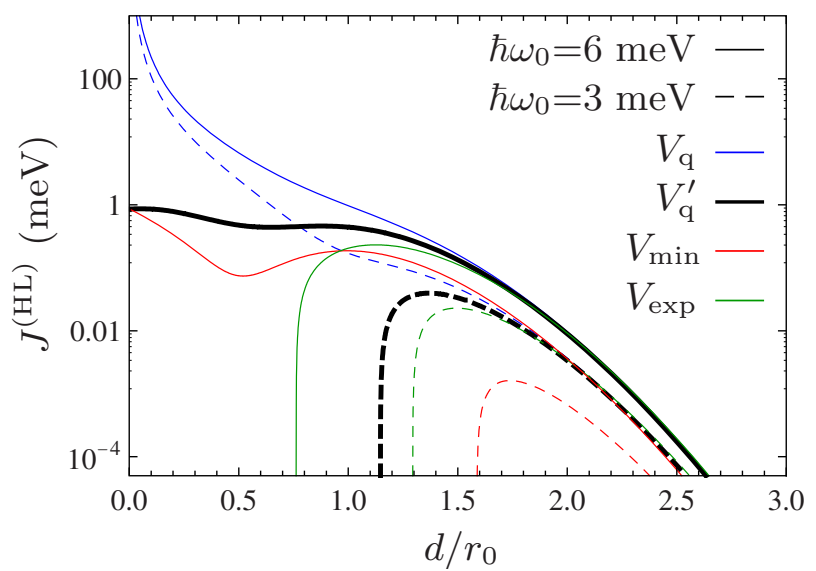

FIG. 2. (Color online) Exchange coupling in the Heitler-London approximation as a function of the interdot distance. Two different confinement strengths $\hbar \omega_{0}$ are used: continuous lines correspond to $\hbar \omega_{0}=6 \mathrm{meV}$ while dashed lines have $\hbar \omega_{0}=3 \mathrm{meV}$.

three other potentials yield (unphysical) negative exchange couplings (near the abrupt decreases of the exchange coupling in the logarithmic plots) within the Heitler-London approximation at short distances and low confinement energies. This behavior is well known for the Heitler-London approximation. ${ }^{11,13}$ Eventually, the Heitler-London approximation also breaks down for the potential $V_{q}$ and predicts a negative exchange coupling, however, only at lower confinement energies (not shown). At large confinement energies, the potentials $V_{\min }$ and $V_{q}^{\prime}$ give nonnegative, finite values of the exchange coupling at short distances.

In Fig. 3, we show results for the exchange coupling in the Hund-Mulliken approximation at zero magnetic field. At large distances, $d \gg r_{0}$, the exchange coupling is again similar for the four potentials. This behavior is further corroborated by the inset of Fig. 3, showing the exchange coupling as function of the bare tunnel coupling $t$. At large distances, where the tunnel coupling is small, a clear $t^{2}$ dependence is

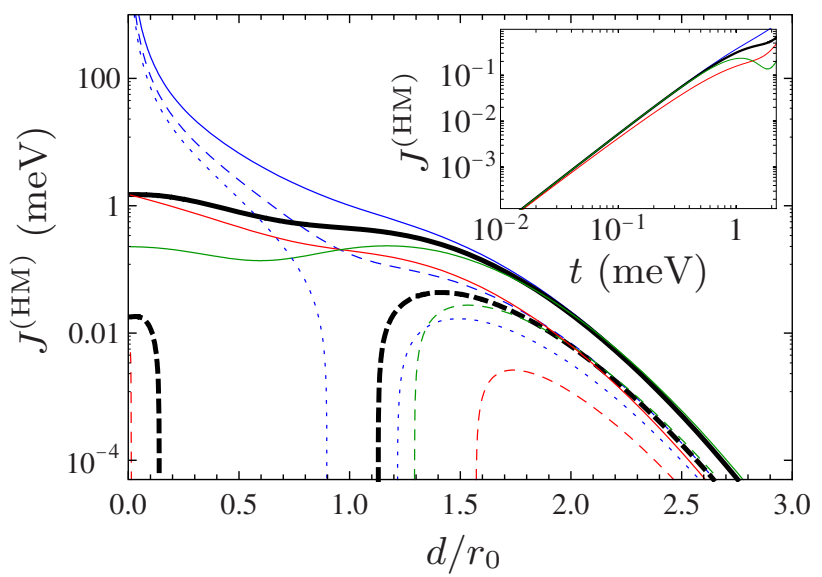

FIG. 3. (Color online) Exchange coupling in the Hund-Mulliken approximation as function of the interdot distance (see Fig. 2 for legend). Two different confinement strengths are used. We also show results for $V_{q}$ with $\hbar \omega_{0}=2 \mathrm{meV}$ (dotted line). In the inset, the exchange coupling as function of the bare tunnel coupling $t$ illustrates the general $t^{2}$ dependence at large interdot distances.

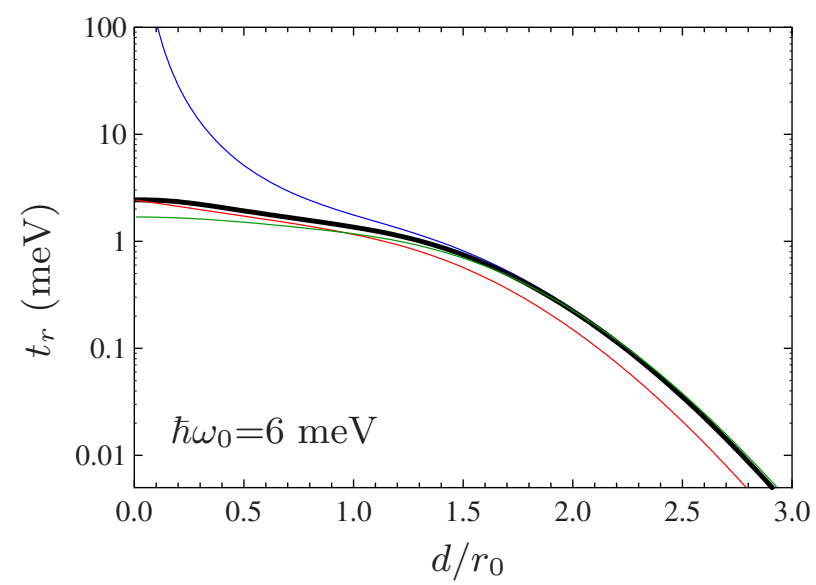

FIG. 4. (Color online) Renormalized tunnel coupling as function of interdot distance (see Fig. 2 for legend).

found as expected in the Hubbard model picture. At short distances, $d \lesssim r_{0}$, and at low confinement energies, $\hbar \omega_{0}$ $=3 \mathrm{meV}$, only the potential $V_{q}$ gives nonnegative values of the exchange coupling. However, at lower confinement energies, $\hbar \omega_{0}=2 \mathrm{meV}$, the Hund-Mulliken approximation also breaks down for this potential around $d=r_{0}$ (dotted line in Fig. 3). At higher confinement energies, $\hbar \omega_{0}=6 \mathrm{meV}$, all potentials yield positive values of the exchange coupling. Also in the Hund-Mulliken approximation, the potential $V_{q}$ results in a diverging exchange coupling at low interdot distances.

In order to explain the observed trends, we consider Eq. (5) for the exchange coupling in the Hund-Mulliken approximation. At large confinement energies, the renormalized tunnel coupling may ensure a well-behaved limit at short interdot distances. At the same time, a too small renormalized tunnel coupling $t_{r}$ can result in negative values of the exchange coupling. In Fig. 4, we show the renormalized tunnel coupling $t_{r}$ as function of the interdot distance. At large interdot distances, the renormalized tunnel coupling is similar for the four potentials, although somewhat smaller for $V_{\min }$.

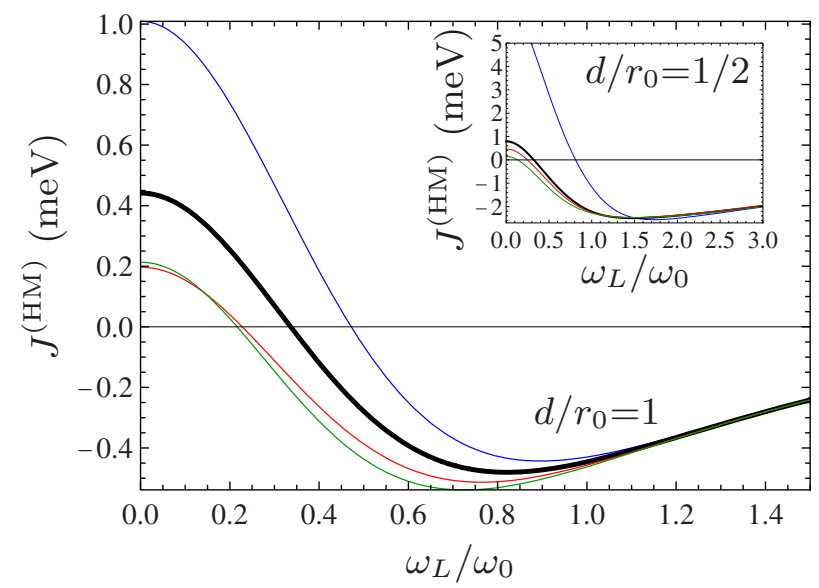

FIG. 5. (Color online) Exchange coupling in the Hund-Mulliken approximation as function of the magnetic field (see Fig. 2 for legend). Results are shown for $\hbar \omega_{0}=6 \mathrm{meV}$ and two different interdot distances. 
The smaller tunnel coupling is due to the height of the barrier separating the two dots being larger for $V_{\min }$ compared to the other potentials. This explains the results for the exchange coupling at large interdot distances within the HundMulliken approximation shown in Fig. 3. At short distances, the potentials $V_{\text {exp }}, V_{\text {min }}$, and $V_{q}^{\prime}$ yield qualitatively identical results with well-behaved limits for the renormalized tunnel coupling and, consequently, also well-behaved values for the exchange couplings in Fig. 3. In contrast, the renormalized tunnel coupling and the exchange energy diverge for $V_{q}$ at short interdot distances. The large renormalized tunnel coupling at intermediate distances ensures positive values of the exchange coupling in the Hund-Mulliken approximations, but eventually leads to a diverging exchange energy at short distances.

We attribute the divergence of the renormalized tunnel coupling for $V_{q}$ to the particular behavior of the potential at short interdot distances. Unlike the three other potentials, the potential $V_{q}$ does not simplify to a single-dot potential at $d$ $=0$ (the potential $V_{\text {exp }}$ already collapses into a single wide dot at $\left.d \lesssim 3 r_{0} / 4\right)$. In fact, it contains a diverging term of the form $x^{4} / d^{2}$. To remedy this problem, we introduced the potential $V_{q}^{\prime}$ with the modified denominator $4 d^{2} \rightarrow 4 d^{2}+x^{2}$. At short interdot distances, we then have $V_{q}^{\prime}(x, 0) \sim\left(x^{2}\right.$ $\left.-d^{2}\right)^{2} /\left(4 d^{2}+x^{2}\right) \rightarrow x^{2}$ corresponding to a single harmonic potential. At large distances, $d \gg r_{0}$, the potential $V_{q}^{\prime}$ is similar to $V_{q}$, while the modified denominator ensures well-behaved limits for the exchange coupling and the renormalized tunnel coupling as illustrated in Figs. 3 and 4.

Finally, we turn to the magnetic field dependence of the exchange coupling. The Hund-Mulliken approximation is typically more reliable than the Heitler-London approximation in predicting the magnetic field dependence. In Fig. 5, we consequently show Hund-Mulliken predications of the exchange coupling as function of the applied magnetic field.
At large magnetic fields, the exchange coupling is similar for the four potentials, whereas different behaviors are seen at low fields, corresponding to the differences seen at zero magnetic field in Fig. 3. At large fields, magnetic compression suppresses the tunnel coupling and we recover the $J \propto t^{2}$ dependence also seen at large interdot distances with zero magnetic field, as illustrated in the inset of Fig. 3. At short interdot distances and small magnetic field, the exchange coupling for $V_{q}$ again blows up due to the diverging term in the potential. In contrast, the behavior of the exchange coupling corresponding to $V_{q}^{\prime}$ is again well behaved at small fields as seen in the inset of Fig. 5.

\section{CONCLUSIONS}

We have studied the exchange coupling between electron spins in double quantum dots within the Heitler-London and Hund-Mulliken approximations using four different confining potentials. At large interdot distances and at high magnetic fields, the exchange coupling is only weakly sensitive to the details of the potentials. In contrast, at short interdot distances, the exchange coupling depends on the choice of potential. At short interdot distances and low magnetic fields, the potential $V_{q}$ yields a diverging exchange coupling. We have slightly modified this potential in order to remedy this problem. The simple expressions for the exchange coupling presented in this work may by useful as guidelines in numerical studies and in the modeling of experimental setups.

\section{ACKNOWLEDGMENTS}

The work by C.F. was supported by the Villum Kann Rasmussen Foundation. A.P.J. is grateful to the FiDiPro program of the Finnish Academy.
${ }^{1}$ D. Loss and D. P. DiVincenzo, Phys. Rev. A 57, 120 (1998).

${ }^{2}$ G. Burkard, D. Loss, and D. P. DiVincenzo, Phys. Rev. B 59, 2070 (1999).

${ }^{3}$ J. R. Petta, A. C. Johnson, J. M. Taylor, E. A. Laird, A. Yacoby, M. D. Lukin, C. M. Marcus, M. P. Hanson, and A. C. Gossard, Science 309, 2180 (2005).

${ }^{4}$ W. A. Coish and D. Loss, Phys. Rev. B 72, 125337 (2005).

${ }^{5}$ X. Hu and S. Das Sarma, Phys. Rev. Lett. 96, 100501 (2006).

${ }^{6}$ M. Stopa and C. M. Marcus, Nano Lett. 8, 1778 (2008).

${ }^{7}$ Q. Li, L. Cywiński, D. Culcer, X. Hu, and S. Das Sarma, Phys. Rev. B 81, 085313 (2010).

${ }^{8}$ B. Szafran, F. M. Peeters, and S. Bednarek, Phys. Rev. B 70, 205318 (2004).

${ }^{9}$ M. Helle, A. Harju, and R. M. Nieminen, Phys. Rev. B 72,
205329 (2005).

${ }^{10}$ L. X. Zhang, D. V. Melnikov, and J. P. Leburton, Phys. Rev. B 74, 205306 (2006).

${ }^{11}$ J. Pedersen, C. Flindt, N. A. Mortensen, and A.-P. Jauho, Phys. Rev. B 76, 125323 (2007).

${ }^{12}$ J. G. Pedersen, L. Zhang, M. J. Gilbert, and J. Shumway, J. Phys.: Condens. Matter 22, 145301 (2010).

${ }^{13}$ M. J. Calderón, B. Koiller, and S. Das Sarma, Phys. Rev. B 74, 045310 (2006).

${ }^{14}$ The ground state of $V_{\text {exp }}$ at large interdot distances cannot be obtained analytically, but we have checked that the Fock-Darwin state is still a good approximation to the ground state found by numerical diagonalization of the corresponding single-particle Hamiltonian in Eq. (3). 